\title{
Liver Stiffness
}

National Cancer Institute

\section{Source}

National Cancer Institute. Liver Stiffness. NCI Thesaurus. Code C138328.

The modulus of elasticity of the liver, representing the resistance of the liver to deformation. Liver stiffness has been shown to be a surrogate marker of advanced fibrosis and cirrhosis. Liver stiffness, measured by transient elastography, is an independent predictor of liver failure, hepatocellular carcinoma, and mortality in cirrhotic patients coinfected with the human immunodeficiency virus or acquired immune deficiency syndrome, and hepatitis C virus. 